\title{
Effect of Compliant Walls on Secondary Instabilities in Boundary-Layer Transition
}

\author{
Ronald D. Joslin† \\ NASA Langley Research Center, Hampton, Virginia 23681 \\ and \\ Philip J. Morrisț \\ Pennsylvania State University, University Park, Pennsylvania 16802
}

Abstract

For aerodynamic and hydrodynamic vehicles, it is highly desirable to reduce drag and noise levels. A reduction in drag leads to fuel savings. In particular for submersible vehicles, a decrease in noise levels inhibits detection. A suggested means to obtain these reduction goals is by delaying the transition from laminar to turbulent flow in external boundary layers. For hydrodynamic applications, a passive device which shows promise for transition delays is the compliant coating. In previous studies with a simple mechanical model representing the compliant wall, coatings were found that provided transition delays as predicted from the semi-empirical $e^{n}$ method. Those studies were concerned with the linear stage of transition where the instability of concern is referred to as the primary instability. For the flat-plate boundary layer, the Tollmien-Schlichting (TS) wave is the primary instability. In one of those studies, it was shown that three-dimensional (3-D) primary instabilities, or oblique waves, could dominate transition over the coatings considered. From the primary instability, the stretching and tilting of vorticity in the shear flow leads to a secondary instability mechanism. This has been theoretical described by Herbert based on Floquet theory. In the present study, Herbert's theory is used to predict the development of secondary instabilities over isotropic and non-isotropic compliant walls. Since oblique waves may be dominant over compliant walls, a secondary theory extention is made to allow for these 3 -D primary instabilities. The effect of variations in primary amplitude, spanwise wavenumber, and Reynolds number on the secondary instabilities are examined. As in the rigid wall case, over compliant walls the subharmonic mode of secondary instability dominates for low-amplitude primary disturbances. Both isotropic and non-isotropic compliant walls lead to reduced secondary growth rates compared to the rigid wall results. For high frequencies, the non-isotropic wall suppresses the amplification of the secondary instabilities, while instabilities over the isotropic wall may grow with an explosive rate similar to the rigid wall results. For the more important lower frequencies, both isotropic and non-isotropic compliant walls suppress the amplification of secondary instabilities compared to the rigid wall results. The twofold major discovery and demonstration of the present investigation are: (1) the use of passive devices, such as compliant walls, can lead to significant reductions in the secondary instability growth rates and amplification; (2) suppressing the primary growth rates and subsequent amplification enable delays in the growth of the explosive secondary instability mechanism.

\section{Introduction}

Research involving flow over flexible walls was started in the late-1950's by Kramer ${ }^{1,2}$. Experimentally, Kramer found significant drag reductions using rubber coatings over rigid walls. Investigators in the 1960 's focused on the task of experimentally duplicating and theoretically explaining Kramer's results. The majority of these studies failed to produce any comparable results; yet, the theoretical results laid the foundation for all future studies involving flexible walls. Interest turned toward the use of compliant walls for turbulent drag reduction. In the 1970's NASA ${ }^{3}$ and in the 1980 's the Office of Naval Research ${ }^{4}$ sponsored investigations involving the use of compliant walls for the turbulent problem. Althought most of the results from this era were either inconclusive or unsatisfactory, the contributions, together with earlier results, have acted as stepping stones to the understanding of the physically complex fluid/wall interaction phenomena. A comprehensive review of the pioneering studies was given by Bushnell, Hefner and Ash', in particular for the turbulent flow problem. More recent reviews were given by Riley, Gad-el-Hak and Metcalfe ${ }^{6}$, Gad-el-Hak ${ }^{7,8}$, and Carpenter ${ }^{9}$.

Motivation for the present investigation is partially derived from the following favorable theoretical and experimental results. In the early 1980's, Carpenter and Garrad $^{10,11}$ showed theoretically that Kramer-type surfaces could lead to potential delays in transition. Further, they indicated deficiencies in previous investigations which may have prevented their achieving results comparable to Kramer's. Only recently, experiments performed by Willis ${ }^{12}$ and Gaster ${ }^{13}$ showed favorable results using compliant walls. As outlined in the above mentioned reviews, a number of investigations in the past ten years have been conducted involving flexible walls. A main emphasis of these studies was to under- 
stand the physical mechanisms involved in the fluid/wall interaction of transitional and turbulent flows. Most of these studies focused on the 2-D instability problem, except Yeo $^{14}$ who showed that a lower critical Reynolds number existed for the isotropic compliant wall for 3-D instability waves. Carpenter and Morris ${ }^{15}$ and Joslin, Morris and Carpenter ${ }^{16}$ have shown that $3-\mathrm{D}$ TollmienSchlichting waves can have greater growth rates over compliant walls than 2-D waves. However, they showed that, even though 3 -D waves may be dominant, transition delays are still obtainable through the use of compliant walls. They considered a compliant wall model used by Grosskreutz ${ }^{17}$ for his turbulent boundary-layer experiments.

In this paper, the growth rates and amplification of secondary instabilities over compliant walls are predicted and compared to the rigid wall results. A theory for secondary instabilities is used which is based on Floquét Theory and was developed by Herbert ${ }^{18}$. This theory agrees remarkably well with experiments, in particular those of Klebanoff, Tidstrom and Sargent ${ }^{19}$ for peak-valley splitting (fundamental) and Kachanov and Levchenko ${ }^{20}$ for peak-valley alignment (subharmonic). The secondary instability theory is extended to allow for 3-D primary instabilities, which are dominant over the compliant walls considered. Appropriate boundary conditions for the Grosskreutz ${ }^{17}$ wall model are derived for the secondary analysis.

In the next section, the primary instability problem is outlined. In section three, the secondary instability problem is discussed. The numerical methods, results, and a summary follow in the remaining sections.

\section{Primary Wave Model}

Results for 2-D and 3-D primary instabilities over compliant and rigid walls have been presented by Joslin $^{21}$, Morris and Carpenter ${ }^{15,16}$. Since the secondary instability theory is based on and includes a primary instability analysis, brief derivations of the dynamic equations and boundary conditions for the 3 -D primary instability problem are included below. The disturbances are represented as travelling waves which may grow or decay as they propagate. Nonlinear coupling is ignored so that individual components of the frequency spectrum may be studied. Additionally, the quasi-parallel assumption is made.

Consider an incompressible laminar, boundary-layer flow over a smooth flat wall. The Navier-Stokes equations govern the flow. The Blasius profile is used to represent the mean flow.

A small-amplitude disturbance is introduced into the laminar flow. A normal mode representation is given as

$$
\begin{aligned}
\left\{v_{1}^{\prime}, \Omega_{1}^{\prime}\right\}(x, y, z, t) & =\left\{v_{1}, \Omega_{1}\right\}(y) \exp [i(x \alpha \cos \phi \\
& +z \alpha \sin \phi-\omega t)]+c . c .
\end{aligned}
$$

where $v_{1}$ and $\Omega_{1}$ are the complex eigenfunctions of normal velocity and vorticity, respectively. To obtain a real solution, complex conjugate solutions denoted by c.c. are required. $\alpha$ is the wavenumber, $\omega$ is the frequency, and $\phi$ is the wave angle. In general, $\alpha$ and $\omega$ are complex leading to an ambiguity in the system. For temporal analyses, $\alpha$ is a real specified wavenumber and $\omega$ is the complex eigenvalue. For spatial analyses, $\omega$ is a real specified frequency and $\alpha$ is the complex eigenvalue. For the compliant wall problem, Joslin, Morris and Carpenter ${ }^{16}$ have shown that the use of eqn. (1) leads to an overestimation of the growth of the wave as it propagates. The wave actually propagates in a nearly streamwise direction which is in the direction of the group velocity, not normal to the wave fronts. In the present paper, the secondary instabilities are investigated using this simple representation of the primary instabilities. Since the present approach is conservative, it should exemplify the benefits of using compliant walls as a means to obtain transition delays. Also, a major emphasis and motivation of the present study is to determine the behavior, or response, of the phenomena-namely secondary instabilities-to compliant walls.

If the normal mode relation (1) is substituted into the linearized form of the Navier-Stokes equations, the following nondimensional system results:

$$
v_{1}^{\prime \prime \prime \prime}+a_{1}(y) v_{1}^{\prime \prime}+a_{2}(y) v_{1}=0
$$

where

$$
\begin{aligned}
a_{1}(y) & =-i R_{\delta}\left(U_{o}(y) \alpha \cos \phi-\omega\right)-2 \alpha^{2} \\
a_{2}(y) & =i R_{\delta} \alpha^{2}\left(U_{o}(y) \alpha \cos \phi-\omega\right) \\
& +i R_{\delta} \alpha \cos \phi U_{o}^{\prime \prime}(y)+\alpha^{4}
\end{aligned}
$$

and

$$
\Omega^{\prime \prime}{ }_{1}+a_{3}(y) \Omega_{1}+a_{4}(y) v_{1}=0
$$

where

$$
\begin{aligned}
& a_{3}(y)=-\alpha^{2}-i R_{\delta}\left(U_{o}(y) \alpha \cos \phi-\omega\right) \\
& a_{4}(y)=-i R_{\delta} \alpha \sin \phi U_{o}^{\prime}(y) .
\end{aligned}
$$

The equations are nondimensionalized using the freestream velocity $U_{\infty}$, kinematic viscosity $\nu$, and an appropriate length scale. Convenient lengths for the boundary-layer scale with the $x$-Reynolds number, $R_{x}=U_{\infty} x / \nu$. These include a thickness, $\delta$, where the Reynolds number is defined $R_{\delta}=R_{x}^{1 / 2}$ and a boundary-layer displacement thickness, $\delta^{*}$, where $R_{\delta^{*}}=$ 
$17207 R_{x}^{1 / 2}$. Eqns. (2) and (3) are referred to as the OrrSommerfeld and Squire equations, respectively. The system requires six boundary conditions. Requiring that the disturbance fluctuations vanish at infinity supplies three:

$$
v_{1}(y), v_{1}^{\prime}(y), \Omega_{1}(y) \rightarrow 0 \quad \text { as } \quad y \rightarrow \infty
$$

The remaining boundary conditions are determined from the compliant wall model.

The compliant wall model used for the present paper was introduced by Grosskreutz ${ }^{17}$ in his experimental drag reduction studies with turbulent boundary layers. He suggested that the link between streamwise and normal surface displacements would cause a negative production of turbulence near the wall. Although his results for the turbulent flow were disappointing, the surface does react to the fluid fluctuations in transitional flow in such a way as to reduce production of instability growth. Carpenter and Morris ${ }^{22}$ have shown by an energy analysis how the many competing energy-transfer mechanisms are influenced by the compliant wall presence. Of note is the reduced energy production by the Reynolds stress which may cause the reduced growth rates. Further, Joslin, Morris and Carpenter ${ }^{16}$ predicted that transition delays of 4-10 times the rigid wall transition Reynolds number were achievable with this coating. So the model has been extended to allow for a secondary instability analysis.

The mechanical model consists of a thin, elastic plate supported by hinged and sprung rigid members inclined to the horizontal and facing upstream at an angle, $\theta$, when in equilibrium. A sketch of the mechanical wall model is shown in Fig. 1. The boundary conditions are obtained by enforcing a balance of forces in the streamwise and spanwise directions and the continuity of fluid and wall motion. These are given below in linearized form.

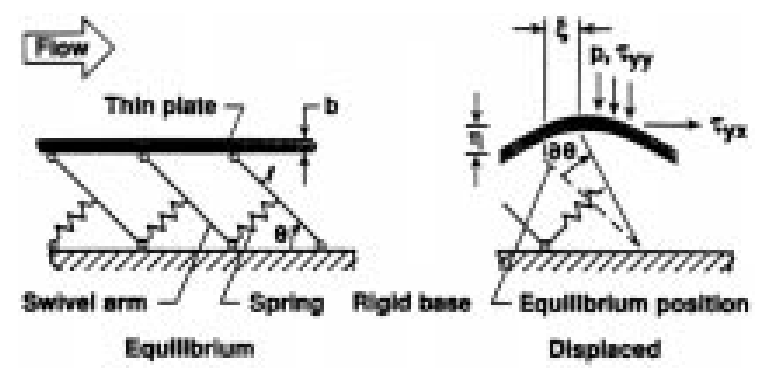

Fig. 1 Mechanical model representing the Grosskreutz compliant coating.
For small displacements of an element out of equilibrium, the mechanical surface can be thought to move in a direction perpendicular to the rigid swivel-arm. The horizontal and vertical displacements $\left(\xi_{1}, \eta_{1}\right)$ are linked to the angular displacement $(\delta \theta)$ by

$$
\xi_{1}=\ell \delta \theta \sin \theta \quad \text { and } \quad \eta_{1}=\ell \delta \theta \cos \theta
$$

where $\ell$ is the length of the rigid-arm member. Equations of motion for the element in the streamwise and spanwise directions may be obtained by a balance of the forces of the fluid fluctuations acting on the surface and the forces due to the wall motion. These equations are

$$
\begin{aligned}
\rho_{m} b \frac{\partial^{2} \eta_{1}}{\partial t^{2}} & +\left(B_{x} \frac{\partial^{4} \eta_{1}}{\partial x^{4}}+2 B_{x z} \frac{\partial^{4} \eta_{1}}{\partial x^{2} \partial z^{2}}\right. \\
& \left.+B_{z} \frac{\partial^{4} \eta_{1}}{\partial z^{4}}\right) \cos ^{2} \theta+K_{E} \eta_{1}-E_{x} b \frac{\partial^{2} \xi_{1}}{\partial x^{2}} \sin \theta \cos \theta \\
& =\left(-p+\tau_{y y}\right) \cos ^{2} \theta+\tau_{y x} \sin \theta \cos \theta
\end{aligned}
$$

and

$$
\rho_{m} b \frac{\partial^{2} \zeta_{1}}{\partial t^{2}}+K_{S} \zeta_{1}-E_{z} b \frac{\partial^{2} \zeta_{1}}{\partial z^{2}}=\tau_{y z}
$$

where

$$
\left\{B_{x}, B_{z}\right\}=\frac{\left\{E_{x}, E_{z}\right\} b^{3}}{12\left(1-\nu_{x} \nu_{z}\right)} \quad \text { and } \quad B_{x z}=\sqrt{B_{x} B_{z}} .
$$

$\zeta_{1}$ is the spanwise surface displacement. $\rho_{m}$ and $b$ are the plate density and thickness; $\left(B_{x}, B_{x z}, B_{z}\right)$ are the flexural rigidities of the plate in the streamwise, transverse, and spanwise directions; $\left(E_{x}, E_{z}\right)$ are the moduli of elasticity of the plate; $K_{E}, K_{S}$ are the effective streamwise and spanwise spring stiffness; $p$ is the pressure fluctuation which is obtained from the fluid momentum equations; and $\tau_{y x}, \tau_{y y}$ and $\tau_{y z}$ are the streamwise, normal, and spanwise viscous shear stress fluctuations in the fluid acting on the wall.

The terms on the left hand side of eqn. (6) refer to mechanical forces and the terms on the right refer to fluid motion forces due to viscous stress and pressure fluctuations. For the case where the ribs are aligned at $\theta=0^{\circ}$, the wall becomes isotropic and reduces to the theoretical model studied by Carpenter and Garrad ${ }^{10,11}$. Otherwise the wall is referred to as non-isotropic and the rib angle is determined by $\theta$.

The continuity of fluid/wall motion is given in the streamwise, normal, and spanwise directions, respectively as

$$
\begin{gathered}
\frac{\partial \xi_{1}}{\partial t}=u_{1}+\eta_{1} U_{o}^{\prime} \\
\frac{\partial \eta_{1}}{\partial t}=v_{1} \\
\frac{\partial \zeta_{1}}{\partial t}=w_{1}
\end{gathered}
$$


where $\left(u_{1}, v_{1}, w_{1}\right)$ are the disturbance velocity components in the streamwise, normal and spanwise directions. For the Grosskreutz coating, $K_{S} \rightarrow \infty$ is assumed, which from eqn. (7) would result in zero effective spanwise surface displacement. This implies from eqn. (10) that $w_{1}(0)=0$. Strictly speaking, if the assumption $K_{S} \rightarrow \infty$ is relaxed, the resulting instabilities have larger growth rates. This suggests that spanwise stiffeners are stabilizing to a disturbed flow. So with the assumption enforced, a better coating for potential transition delays results. The surface displacement takes the same normal mode form as the primary wave given by eqn. (1). The normal modes are substituted into eqns. (6-10). The equations can be reduced to three equations in terms of the normal velocity and vorticity ${ }^{16,21}$.

\section{Secondary Instability Theory}

In this section, a secondary instability theory developed by Herbert ${ }^{18}$ is extended to allow for 3-D primary instabilities. Additionally, boundary equations describing the compliant walls are introduced for secondary instabilities. The flow is governed by the Navier-Stokes equations. Instantaneous velocity and pressure components are introduced and given as

$$
\begin{aligned}
& \bar{v}(\tilde{x}, y, \tilde{z}, t)=\bar{v}_{2}(\tilde{x}, y, \tilde{z}, t)+B \bar{v}_{3}(\tilde{x}, y, \tilde{z}, t) \\
& p(\tilde{x}, y, \tilde{z}, t)=p_{2}(\tilde{x}, y, \tilde{z}, t)+B p_{3}(\tilde{x}, y, \tilde{z}, t)
\end{aligned}
$$

where $p_{3}$ and $\bar{v}_{3}=\left(u_{3}, v_{3}, w_{3}\right)$ are the secondary disturbance pressure and velocity in the fixed laboratory reference frame $(\tilde{x}, y, \tilde{z})$; and $p_{2}$ and $\bar{v}_{2}=\left(u_{2}, v_{2}, w_{2}\right)$ are the basic pressure and velocity given by,

$$
\begin{aligned}
& \bar{v}_{2}(\tilde{x}, y, \tilde{z}, t)=\left\{U_{o}(y), 0,0\right\}+A\left\{u_{1}, v_{1}, w_{1}\right\}(\tilde{x}, y, \tilde{z}, t) \\
& p_{2}(\tilde{x}, y, \tilde{z}, t)=A p_{1}(\tilde{x}, y, \tilde{z}, t)
\end{aligned}
$$

The basic flow is given by the Blasius profile and eigenfunctions of the primary wave. Assume locally that the primary wave is periodic in t and periodic in $(\tilde{x}, \tilde{z})$ with wavelength $\lambda_{r}=2 \pi / \alpha_{r}$ and efine a disturbance phase velocity which is

$$
\bar{c}_{r}=\left(c_{x}=\omega_{r} / \alpha_{r} \cos \phi, 0, c_{z}=\omega_{r} / \alpha_{r} \sin \phi\right) .
$$

Then in a frame moving with the primary wave,

$$
\bar{v}_{1}(\tilde{x}, y, \tilde{z})=\bar{v}_{1}(x, y, z)=\bar{v}_{1}\left(x+\lambda_{x}, y, z+\lambda_{z}\right)
$$

where $(x, z)$ is the reference frame moving with the wave. With an appropriate normalization of primary eigenfunctions $\left(u_{1}, v_{1}, w_{1}\right)$ the amplitude, $A$, directly measures the maximum streamwise rms fluctuation. This is given by

$$
\max _{0<y<\infty}\left|u_{1}(y)\right|^{2}=\left|u_{1}\left(y_{m}\right)\right|^{2}=1 / 2
$$

The instantaneous velocities and pressure (11) are substituted into the Navier-Stokes equations which are linearized with respect to the secondary amplitude, $B$. The disturbance pressure is eliminated, resulting in the vorticity equations.

$$
\begin{aligned}
{\left[\frac{1}{R_{\delta}} \nabla^{2}-\frac{\partial}{\partial t}\right] \bar{\Omega}_{3}-\left(\bar{v}_{2} \cdot \nabla\right) \bar{\Omega}_{3}-\left(\bar{v}_{3} \cdot \nabla\right) \bar{\Omega}_{2} } \\
+\left(\bar{\Omega}_{2} \cdot \nabla\right) \bar{v}_{3}+\left(\bar{\Omega}_{3} \cdot \nabla\right) \bar{v}_{2}=0
\end{aligned}
$$

with the continuity equation

$$
\nabla \cdot \bar{v}_{3}=0
$$

As with the primary problem, the equations are formed in terms of the normal velocity $\left(\bar{v}_{3}\right)$ and vorticity $\left(\bar{\Omega}_{3}\right)$.

The equations are found to take the form ${ }^{21}$,

$$
\begin{gathered}
{\left[\frac{1}{R_{\delta}} \nabla^{2}-\frac{\partial}{\partial t}-\left(U_{o}-c_{x}\right) \frac{\partial}{\partial x}+c_{z} \frac{\partial}{\partial z}\right] \Omega_{3}} \\
-\frac{d U_{0}}{d y} \frac{\partial v_{3}}{\partial z}+A\left\{-\left(\bar{v}_{1} \cdot \nabla\right) \Omega_{3}-\left(\bar{v}_{3} \cdot \nabla\right) \Omega_{1}\right. \\
+\left(\epsilon_{1}+\frac{\partial v_{1}}{\partial z}\right) \frac{\partial v_{3}}{\partial x}+\Omega_{1} \frac{\partial v_{3}}{\partial y}+\left(\rho_{1}-\frac{\partial v_{1}}{\partial x}\right) \frac{\partial v_{3}}{\partial z} \\
\left.-\frac{\partial v_{1}}{\partial z} \frac{\partial u_{3}}{\partial y}+\frac{\partial v_{1}}{\partial x} \frac{\partial w_{3}}{\partial y}\right\}=0
\end{gathered}
$$

and,

$$
\begin{aligned}
& {\left[\frac{1}{R_{\delta}} \nabla^{2}-\frac{\partial}{\partial t}-\left(U_{0}-c_{x}\right) \frac{\partial}{\partial x}+c_{z} \frac{\partial}{\partial z}\right] \nabla^{2} v_{3}+\frac{d^{2} U_{o}}{d y^{2}} \frac{\partial v_{3}}{\partial x}} \\
& +A\left\{\left[-\left(\bar{v}_{1} \cdot \nabla\right) \nabla^{2}-\frac{\partial}{\partial y} \nabla^{2} v_{1}-\left(\frac{\partial^{2} u_{1}}{\partial x^{2}}+\frac{\partial^{2} u_{1}}{\partial z^{2}}-\frac{\partial^{2} u_{1}}{\partial y^{2}}\right.\right.\right. \\
& \left.+2 \frac{\partial^{2} v_{1}}{\partial x \partial y}\right) \frac{\partial}{\partial x}+\left(\frac{\partial^{2} v_{1}}{\partial z^{2}}-\frac{\partial^{2} v_{1}}{\partial x^{2}}+\frac{\partial^{2} v_{1}}{\partial y^{2}}+2 \frac{\partial^{2} u_{1}}{\partial x \partial y}\right) \frac{\partial}{\partial y} \\
& +\left(\frac{\partial^{2} w_{1}}{\partial y^{2}}-\frac{\partial^{2} w_{1}}{\partial x^{2}}+\frac{\partial^{2} u_{1}}{\partial x \partial z}-\frac{\partial^{2} v_{1}}{\partial y \partial z}\right) \frac{\partial}{\partial z}+\left(\frac{\partial v_{1}}{\partial y}+2 \frac{\partial u_{1}}{\partial x}\right) \\
& \left(\frac{\partial^{2}}{\partial z^{2}}-\frac{\partial^{2}}{\partial x^{2}}+\frac{\partial^{2}}{\partial y^{2}}\right)-2 \frac{\partial v_{1}}{\partial x} \frac{\partial^{2}}{\partial x \partial y}-2\left(\frac{\partial u_{1}}{\partial z}+\frac{\partial w_{1}}{\partial x}\right) \frac{\partial^{2}}{\partial x \partial z} \\
& \left.-\frac{\partial v_{1}}{\partial z} \frac{\partial^{2}}{\partial y \partial z}\right] v_{3}+\left[-\frac{\partial}{\partial x}\left(\nabla^{2} v_{1}\right)+2\left(\frac{\partial^{2} v_{1}}{\partial z^{2}}-\frac{\partial^{2} v_{1}}{\partial x^{2}}+\frac{\partial^{2} v_{1}}{\partial y^{2}}\right.\right. \\
& \left.+2 \frac{\partial^{2} u_{1}}{\partial x \partial y}\right) \frac{\partial}{\partial x}+2\left(\frac{\partial^{2} w_{1}}{\partial x \partial y}-\frac{\partial^{2} v_{1}}{\partial x \partial z}\right) \frac{\partial}{\partial z}-\frac{\partial v_{1}}{\partial x}\left(\frac{\partial^{2}}{\partial x^{2}}+\frac{\partial^{2}}{\partial z^{2}}\right. \\
& \left.-\frac{\partial^{2}}{\partial y^{2}}\right)+2\left(\frac{\partial v_{1}}{\partial y}+2 \frac{\partial u_{1}}{\partial x}\right) \frac{\partial^{2}}{\partial x \partial y}+2 \frac{\partial w_{1}}{\partial x} \frac{\partial^{2}}{\partial y \partial z} \\
& \left.+\frac{\partial v_{1}}{\partial z} \frac{\partial^{2}}{\partial x \partial z}\right] u_{3}+\left[-\frac{\partial}{\partial z}\left(\nabla^{2} v_{1}\right)+2\left(\frac{\partial^{2} u_{1}}{\partial y \partial z}-\frac{\partial^{2} v_{1}}{\partial x \partial z}\right) \frac{\partial}{\partial x}\right. \\
& \left.\left.-\frac{\partial v_{1}}{\partial z}\left(\frac{\partial^{2}}{\partial x^{2}}-\frac{\partial^{2}}{\partial y^{2}}\right)+2 \frac{\partial u_{1}}{\partial z} \frac{\partial^{2}}{\partial x \partial y}\right] w_{3}\right\}=0
\end{aligned}
$$

The disturbance quantities $v_{3}, \Omega_{3}$ and $\partial v_{3} / \partial y$ are required to vanish far from the wall and at the wall for the 
rigid wall case. The compliant wall equations give the remaining boundary conditions in the compliant case. Additionally, the primary amplitude, $A$, is a parameter in the equations and is assumed to be locally non-varying. As $A \rightarrow 0$ the Orr-Sommerfeld and Squire equations result. For the case of interest where $A \neq 0$, the primary eigenfunctions $\left(u_{1}, v_{1}, w_{1}\right)$ appear in the equations as coefficients.

To solve the secondary problem, a normal mode solution having the following form is assumed,

$$
v_{3}(x, y, z, t)=e^{\sigma t+i \beta(z \cos \phi-x \sin \phi)} V(x, y, z)
$$

where $\beta=2 \pi / \lambda_{z}$ is a specified spanwise wavenumber and $\sigma=\sigma_{r}+i \sigma_{i}$ is a temporal eigenvalue or is specified for spatial analyses. $V(x, y, z)$ is a function that represents the class of secondary modes. Floquét theory suggests the form of solution for periodic systems. For the present problem, this may be written

$$
V(x, y, z)=e^{\gamma(x \cos \phi+z \sin \phi)} \tilde{V}(x, y, z)
$$

where $\gamma=\gamma_{r}+i \gamma_{i}$ is the characteristic exponent and $\tilde{V}(x, y, z)$ is periodic in the $(x, z)$ plane and may be represented by a Fourier series. Thus the representation of the secondary instability for a 3 -D basic flow is,

$$
\begin{array}{r}
v_{3}=e^{\sigma t+i \beta(z \cos \phi-x \sin \phi)+\gamma(x \cos \phi+z \sin \phi)} \\
\sum_{n=-\infty}^{\infty} \hat{v}_{n}(y) e^{i(n / 2) \alpha_{r}(x \cos \phi+z \sin \phi)}
\end{array}
$$

This suggests a form of solution for the secondary disturbance based on a coordinate system oriented at an angle $\phi$ with respect to the mean flow and moving with the primary wave. If the coordinate system is aligned with the primary wave, or $\phi=0^{\circ}$, then the solution for the secondary disturbance would follow Herbert, Bertolotti and Santos $^{23}$ who considered a 2-D primary wave.

If solutions given by eqn. (21) are substituted into eqns. (17) and (18), an infinite system of ordinary differential equations result. The dynamic equations are determined by collecting terms in the governing equations with like exponentials. The system consists of two distinct classes of solution because the even and odd modes decouple. Even modes correspond to the fundamental mode of secondary instability, and the odd modes are the subharmonic mode. Only a few terms of the Fourier series are retained since, as shown by Herbert, Bertolotti and Santos ${ }^{23}$, this provides a sufficiently accurate approximation for a $2-\mathrm{D}$ disturbance.

This form of solution indicates two complex quantities, $\sigma$ and $\gamma$, which leads to an ambiguity similar to that found with the Orr-Sommerfeld/Squire problem. There are four unknowns, $\sigma_{r}, \sigma_{i}, \gamma_{r}, \gamma_{i}$. Two can be determined while two must be chosen in some other way.
In the present study, temporally-growing tuned modes are examined. The temporal growth rate is $\sigma_{r}$, and $\sigma_{i}$ can be interpreted as a shift in frequency. In this case, $\gamma_{r}=\gamma_{i}=0$. If $\sigma_{i}=0$, then the secondary disturbance is travelling synchronously with the basic flow.

The boundary conditions for the secondary disturbance are given as

$$
\hat{v}_{n}, \hat{v}_{n}^{\prime}, \hat{\Omega}_{n} \rightarrow 0 \quad \text { as } \quad y \rightarrow \infty
$$

along with the compliant wall boundary conditions. In the rigid wall case,

$$
\hat{v}_{n}, \hat{v}_{n}^{\prime}, \hat{\Omega}_{n}=0 \quad \text { at } \quad y=0
$$

The analysis for the compliant boundary conditions for secondary instabilities follows the same route as was taken for the primary instabilities, except a number of additional terms arise due to the presence of the primary wave.

The fluid/wall motion must be continuous in each direction. In addition, the equations of force $(6,7)$ must balance in the streamwise and spanwise directions in the reference frame moving with the primary wave. Consistent with the fluid equations, the amplitude of the primary wave is assumed to be locally non-varying. In deriving the final form of the wall equations, a significant difference between the primary and secondary form arises from the pressure contribution. The pressure for the secondary disturbance is determined from the momentum equations which are complicated by primary coupling terms.

The continuity of motion between the fluid and solid is given by

$$
\begin{gathered}
\frac{\partial \xi_{3}}{\partial t}=u_{3}+\eta_{3} U_{o}^{\prime}+A\left\{\left(\bar{\xi}_{1} \cdot \nabla\right) u_{3}+\left(\bar{\xi}_{3} \cdot \nabla\right) u_{1}\right\} \\
\frac{\partial \eta_{3}}{\partial t}=v_{3}+A\left\{\left(\bar{\xi}_{1} \cdot \nabla\right) v_{3}+\left(\bar{\xi}_{3} \cdot \nabla\right) v_{1}\right\} \\
\frac{\partial \zeta_{3}}{\partial t}=w_{3}+A\left\{\left(\bar{\xi}_{1} \cdot \nabla\right) w_{3}+\left(\bar{\xi}_{3} \cdot \nabla\right) w_{1}\right\}
\end{gathered}
$$

where

$$
\bar{\xi} \cdot \nabla=\xi \frac{\partial}{\partial x}+\eta \frac{\partial}{\partial y}+\zeta \frac{\partial}{\partial z}
$$

Equations (24-26) involve six unknowns for the velocity fluctuation and surface displacement in a highly coupled system. As with the primary boundary conditions, it is possible to derive a set of equations which represent the surface motion in terms of the normal velocity and vorticity only. This is algebraically very tedious. A complete derivation is given by $\mathrm{Joslin}^{21}$. Note that if $A=0$ in the secondary wall equations, the primary wall equations result. This occurred with the fluid equations as well. 


\section{Numerical Methods of Solution}

The algebraic complexity of the dynamic equations for the secondary disturbance and the compliant wall equations requires that care be taken in applying any numerical technique. Because no theoretical or experimental data are available for the compliant problem, both shooting ${ }^{16}$ and spectral ${ }^{21}$ approximations are used. Also, noting that Bertolotti ${ }^{24}$ has shown for the rigid wall problem with a 2-D primary instability that, after the transformation from spatial to temporal, the solutions are in good agreement, a temporal analysis is presented in this paper.

For the spectral method, Chebyshev series are introduced to approximate each mode of the Fourier series. An algebraic transformation is used to change from the Chebyshev spectral domain $[-1,1]$ to the physical domain. Due to the properties of the Chebyshev polynomial, the equations are recast in integral form. Chebyshev polynomials are used to represent the basic flow in the series which are substituted into the integral equations. For the basic flow, 35 polynomials provided sufficient resolution of the eigenfunctions. The series representing the secondary instability requires 40 polynomials for sufficient convergence to the dominant eigenvalue. For the shooting method, beginning with the equations for the compliant wall, integrations of the disturbance equations across the boundary layer are performed using a Runge-Kutta scheme. At the edge of the boundary layer, the numerical solution vectors are matched with the asymptotic solutions. A very accurate initial guess is found to be required for convergence using this method. To demonstrate the accuracy of the numerical techniques, a comparison for the rigid wall case is made with Herbert ${ }^{25}$ for $R_{\delta}=826.36, F r=83, \beta=0.18$, and $A=0.02$. Herbert obtained the dominant mode $\sigma=0.01184$. In good agreement, the present spectral and shooting methods lead to $\sigma=0.011825$ and $\sigma=0.011839$, respectively.

\section{Results}

For all of the results that follow, the freestream velocity is $20 \mathrm{~m} / \mathrm{s}$, the density is $1000 \mathrm{~kg} / \mathrm{m}^{3}$, and the kinematic viscosity is $1 \times 10^{-6} \mathrm{~m}^{2} / \mathrm{s}$. The coatings considered consist of both isotropic and non-isotropic walls. Both walls were optimized at $R_{\delta^{*}}=2240$ for 2-D primary instabilities. The isotropic wall has properties $\theta=0^{\circ}$, $b=0.735 \mathrm{~mm}, E_{x}=1.385 \mathrm{MN} / \mathrm{m}^{2}, K=0.354 \mathrm{GN} / \mathrm{m}^{3}$ and $\rho_{m}=1000 \mathrm{~kg} / \mathrm{m}^{3}$; and the non-isotropic wall has properties $\theta=60^{\circ}, b=0.111 \mathrm{~mm}, E_{x}=0.509 \mathrm{MN} / \mathrm{m}^{2}$, $K=0.059 \mathrm{GN} / \mathrm{m}^{3}$ and $\rho_{m}=1000 \mathrm{~kg} / \mathrm{m}^{3}$. A Reynolds number of 2240 was chosen because, for a boundary layer over a rigid wall, the disturbance with the critical frequency (in the $e^{n}$ sense) reaches its maximum growth rate near this value of Reynolds number. Accordingly, this is a good choice of Reynolds number for optimizing the wall properties. In considering $3-\mathrm{D}$ instabilities, the walls optimized for 2-D instabilities are used with the addition of isotropic plates. The properties of an isotropic plate are direction independent; that is, $E_{x}=E_{z}$. Although complete details of the optimization process and philosophy are given by Carpenter and Morris ${ }^{22}$, a recap follows.

With a flexible wall present, other modes of instability arise. With changes in the compliant wall properties, stable, or marginally stable, fluid and wall modes can become unstable and dominant. The present wall properties were varied to achieve an optimal specified condition. This desired condition was to achieve a minimum growth rate for a dominant 2-D Tollmien-Schlichting instability while keeping other modes marginally stable. For the secondary analysis, these "optimal" compliant walls led to no additional unstable modes. However, this is not to say that additional growing modes may not appear for different wall properties.

In this analysis, the primary wave amplitude $(A)$ and the secondary instability spanwise wavenumber $(\beta)$ are parameters of the problem. Herbert ${ }^{26}$ showed in his boundary-layer studies for the 2-D primary wave over a rigid wall that as the amplitude increases the growth rate of the secondary instability increases. Also, as the spanwise wavenumber is varied, the temporal growth rate reaches a maximum for a particular wavenumber. Additionally, Herbert showed that the subharmonic mode reaches greater growth rates than the fundamental mode for low amplitude disturbances. These findings were verified by the direct numerical simulations of Spalart and Yang ${ }^{27}$. Althought both the subharmonic and fundamental modes over the compliant walls were examined, emphasis is placed on the subharmonic mode, since as both theory and computations indicate, subharmonic disturbances are more unstable than fundamental disturbances for small amplitudes. Limited fundamental disturbance results are included to verify that these modes do not become the dominant instability over compliant walls.

Primary waves with frequencies that give maximum disturbance growth rates are considered. For the isotropic wall, the maximum growth rate occurs at a frequency $\omega=0.065(F r=\simeq 29.0)$, where $F r=\omega / R \times 10^{6}$. Figure 2 shows the growth rates of the subharmonic and fundamental disturbances as a function of the spanwise wavenumber for the rigid wall and isotropic compliant wall. As the figure shows, growth rates over the compliant wall are reduced in comparison with the rigid wall results over the whole range of spanwise wavenumbers. Additionally, the subharmonic disturbance has much larger growth rates than the fundamental disturbance, as expected. Similar trends are found in the com- 
parison of non-isotropic and rigid wall results. In considering reductions in the growth rates of the subharmonic mode as a result of compliant walls, the isotropic wall suppressed the maximum growth rate by $20 \%$. For the maximum growth rate over the non-isotropic wall, which occurs at a difference frequency than the isotropic wall case, the non-isotropic wall led to a reduction of $17 \%$ compared to rigid wall results. So for a fixed Reynolds number and primary wave amplitude, both isotropic and non-isotropic compliant walls lead to reduced secondary instability growth rates compared to the rigid wall results.

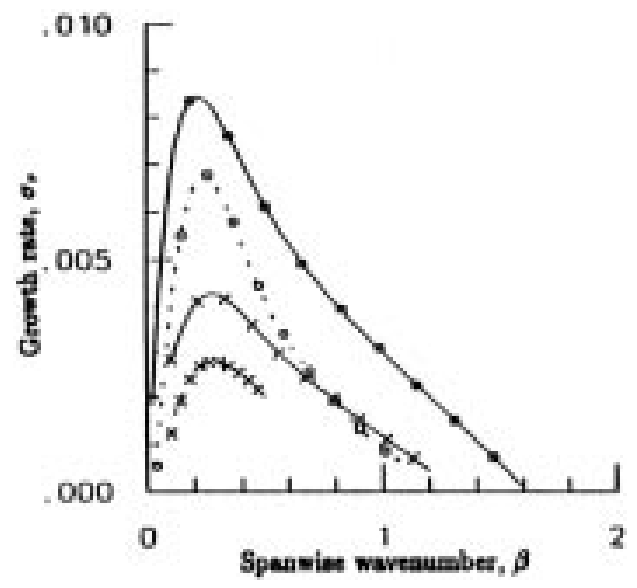

Fig. 2 Growth rates of the secondary instabilities as a function of spanwise wavenumber for $R_{\delta^{*}}=2240, F r \simeq 29.0$, and $A=0.01$. subharmonic: -o-, rigid wall; $\cdots o \cdots$, isotropic wall and fundamental: $-\times-$, rigid wall; $\cdots \times \cdots$, isotropic wall.

A more revealing measure of the effectiveness of using compliant walls to suppress secondary instabilities is to compute the amplitude growth and decay with downstream distance. The amplification of the primary and secondary instabilities are governed by

$$
\ln \frac{A}{A_{0}}=\int_{x_{0}}^{x}-\alpha_{i}^{*} d x \text { and } \ln \frac{B}{B_{o}}=\int_{x_{o}}^{x} \frac{\sigma_{r}^{*}}{c_{r}^{*}} d x
$$

where $A_{o}, B_{0}$ are the initial amplitudes at $x_{o}, A$ and $B$ are the amplitudes at a downstream distance $x$, and $*$ denotes dimensional quantities. As shown by Herbert ${ }^{18}$, the theoretical prediction of primary and secondary amplification by eqns. (27) compares well with the experiments of Kachanov and Levchenko ${ }^{20}$. A similar comparison is shown in Fig. 3, where the theoretical results were obtained with the present numerical techniques.

Before computing similar amplification results over the compliant walls, inferences of the secondary instability growth may be drawn from primary instability results. Figure 4 shows the maximum amplification of various frequency primary waves propagating over the rigid and compliant walls along with the amplification of waves at a frequency $F r=53$. At this frequency, primary amplitudes over the the isotropic wall are similar to those over the rigid wall. This suggests that the development of secondary instabilities might also be similar, since secondary disturbances are parametrically dependent on the basic flow. For the non-isotropic wall results shown in Figure 4, the primary amplitudes are suppressed significantly compared to the rigid wall and isotropic wall cases. Therefore, the non-isotropic wall would likely lead to a very different secondary instability development, most probably with reduced amplitudes. Again from Figure 4, one might expect greater differences in the secondary instability development over both compliant walls as the Reynolds numbers increase and corresponding frequencies decrease.

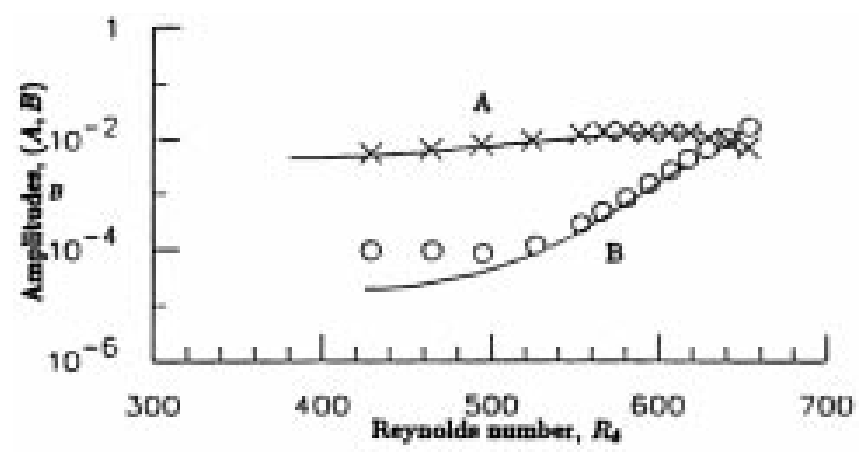

Fig. 3 Amplitude growth as a function of Reynolds number for the subharmonic mode (B) of a 2-D primary wave (A) over a rigid wall at $F r=124, A_{\circ}=0.0044, B_{0}=1.86 \times 10^{-5}$, and $\bar{b}=0.33$. - theory and $(\times, o)$, Kachanov and Levchenko [15].

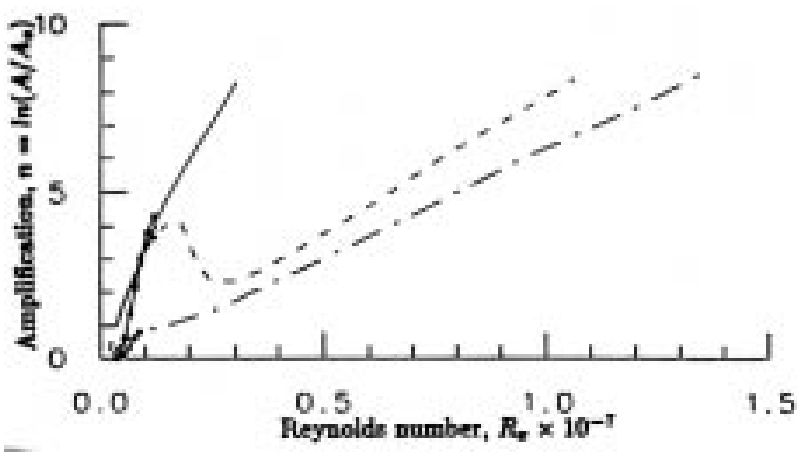

Fig. 4 2-D curves of maximum amplification for TSI waves over a - , rigid wall; --- , isotropic wall; and $-\cdot-$, non-isotropic wall and $-\bullet-$, waves of $F r \simeq 53$. 
To demonstrate these postulations for secondary instabilities, eqns. (27) are used to compute the amplification of primary and secondary instabilities over the rigid, isotropic, and non-isotropic walls for $A_{0}=0.004$, $B_{o}=1 \times 10^{-5}$, and $\bar{b}=0.15$, where $\bar{b}=\beta / R \times 10^{3}$. The initial amplitudes are somewhat arbitrary: the present values were selected to be close to the experiments of Kachanov and Levchenko ${ }^{20}$ for rigid walls. The spanwise wavenumber $(\bar{b})$ was chosen near the maximum growth rate of the secondary instability at the branches of the neutral curve for the rigid wall case. Both primary and secondary amplifications are shown in Fig. 5. Clearly, the results of the secondary instability growth over the isotropic and rigid walls are similar, as postulated, while the non-isotropic wall significantly suppresses the secondary instability growth.

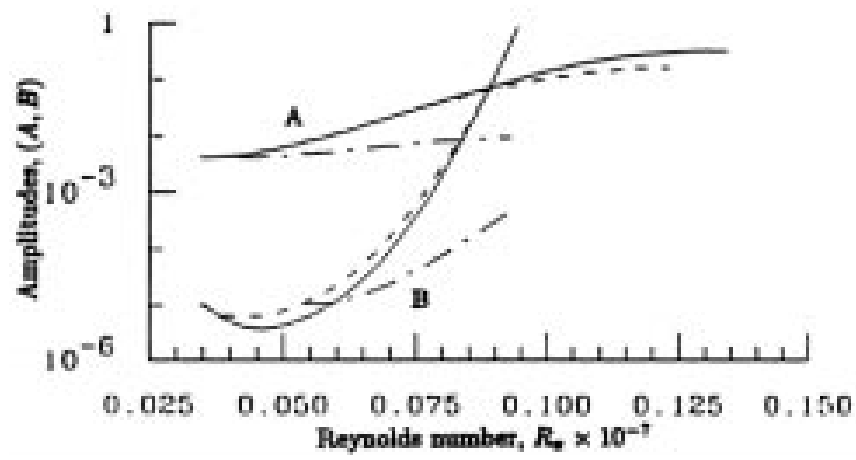

Fig. 5 Amplitude growth as a function of Reynolds number for the subharmonic mode (B) of a 2-D primary wave (A) at $F r \simeq 53, A_{0}=0.004$, $B_{0}=1 \times 10^{-5}$, and $\bar{b}=0.15$ over a - , rigid wall; --- , isotropic wall; and.-- , non-isotropic wall.

The growth of the secondary instability is dependent on the parameters of the basic flow, most probably the primary instability amplitude $(A)$. For example, Fig. 6 shows the amplification of the primary and secondary instabilities over the rigid wall with properties as before and over the non-isotropic wall with both $A_{0}=0.004$ and $A_{o}=0.008$. Even by doubling the initial amplitude of the primary disturbance, the growth of the secondary instability over the non-isotropic wall continues to be suppressed and has not exceeded the primary amplitude upon crossing the neutral curve. Yet, the doubled initial amplitude $\left(A_{0}=0.008\right)$ results in a significant increase in the secondary instability growth compared with the lower amplitude $\left(A_{0}=0.004\right)$ results. Hence, the suppression of the primary instability amplitude is of utmost importance to suppress the onset of the secondary instability growth.

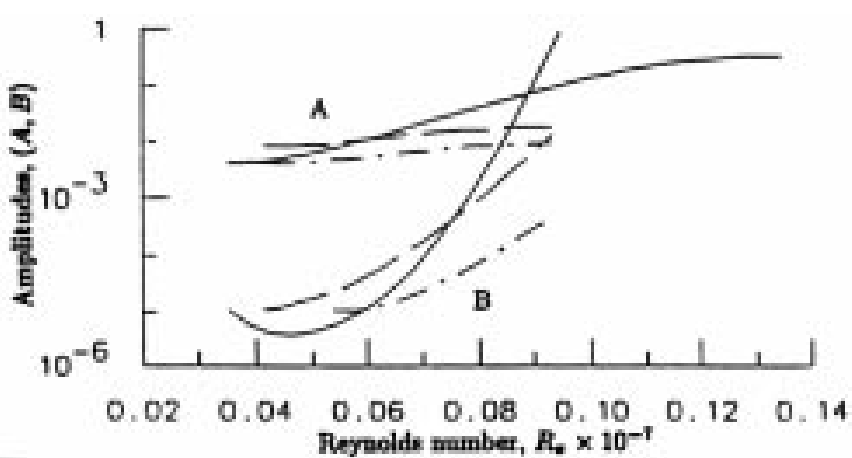

Fig. 6 Amplitude growth as a function of Reynolds number for the subharmonic mode (B) of a 2-D primary wave (A) at $F r \simeq 53, B_{0}=$ $1 \times 10^{-5}$, and $\bar{b}=0.15$ over a with $A_{0}=0.004 ;-\cdot-$, non-isotropic wall with $A_{o}=0.004$; and - , non-isotropic wall with $A_{0}=0.008$.

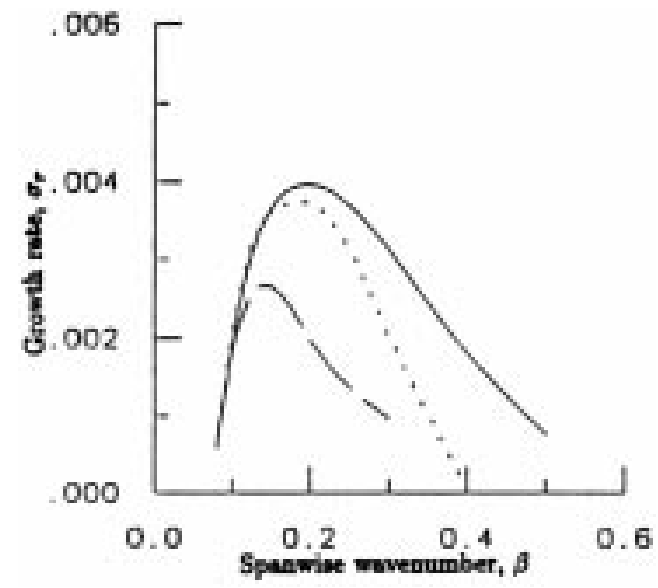

Fig. 7 Growth rates of the subharmonic disturbance for 2-D and 3-D primary waves over the isotropic wall as a function of spanwise wavenumber for $R_{\delta^{*}}=2240, F r \simeq 22.3$, and $A=0.01$. $\phi=0^{\circ} ; \cdots, \phi=10^{\circ}$; and,$- \phi=20^{\circ}$.

Proceeding with investigating the effect of compliant walls on secondary instabilities, 3-D primary waves are introduced and are determined by the specified wave angle $(\phi)$. In Fig. 7, subharmonic disturbance growth rates over the isotropic compliant wall are shown with variation in spanwise wavenumber and primary wave angle $(\phi)$. A frequency $\omega=0.05(F r \simeq 22.3)$ is selected since the $3-\mathrm{D}$ primary wave growth rate is maximized at this frequency for the Reynolds number $R_{\delta^{*}}=2240$. Subharmonic growth rates arising from the 2-D primary wave $\left(\phi=0^{\circ}\right)$ are clearly larger than those from the 3 -D waves. As the primary wave angle $(\phi)$ increases, the subharmonic growth rates continually become more damped. Additionally, for oblique waves $\left(\phi \neq 0^{\circ}\right)$ the secondary disturbances no longer travel synchronously with the primary wave. This is shown in Fig. 8 by the fre- 
quency shifts that result over the isotropic wall. Similar results occur for the non-isotropic wall. It is likely that this shift leads to a reduced efficiency of energy transfer from the basic flow to the secondary disturbance. This frequency shift is as much a result of the $3-\mathrm{D}$ nature of the basic flow as it is of the compliant wall influence. Results similar to those of Fig. 8 were found by Balachandar, Streett and Malik ${ }^{28}$ for the rotating-disk problem which also has a $3-\mathrm{D}$ basic flow.

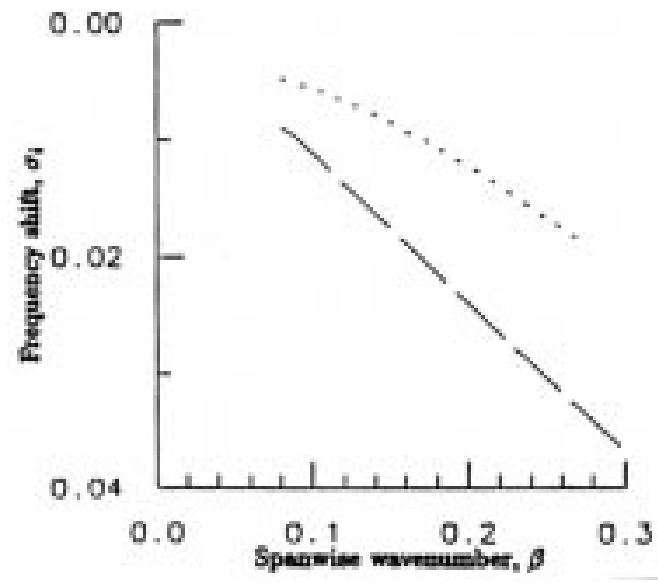

Fig. 8 Frequency shift of the subharmonic disturbance for 3-D primary waves over the isotropic wall as a function of spanwise wavenumber for $R_{\delta *}=2240, F r \simeq 22.3$, and $A=0.01$. $\cdots, \phi=10^{\circ}$ and,$- \phi=20^{\circ}$.

The secondary growth rate comparison in Fig. 7 is misleading as a result of holding the primary amplitude $(A)$ fixed. With a variation in wave angle $(\phi)$, corresponding primary wave amplitudes result as shown by Joslin, Morris and Carpenter ${ }^{16}$. Then a more realistic comparison of secondary growth rates arising from 2-D and 3 -D primary waves should involve amplitudes suited to a given wave angle $(\phi)$. One means to derive these amplitudes is through the use of eqns. (27). In Fig. 9, a comparison of the subharmonic growth rates over the isotropic compliant wall is made for 2-D and 3-D primary waves allowing for amplitude differences. Similar results are found for the non-isotropic wall as well. As shown, the 3 -D primary wave does lead to much larger growth rates than a $2-D$ wave for the compliant walls considered. This clearly demonstrates that in spite of the loss of synchronization with the basic flow, 3-D primary waves lead to dominant secondary instabilities over compliant walls. Again, the amplitude of the primary wave is of utmost importance for determining the secondary disturbance growth. Yet, compliant walls do lead to a reduction in secondary growth rates compared with those for the rigid wall. Also, recall that the amplitudes for 3-D primary waves used in these calculations were determined by a normal mode assumption which leads to a conservative estimation of the primary amplitude ${ }^{16}$, and a more realistic lower amplitude would lead to secondary growth rates somewhere between the $2-\mathrm{D}$ and 3 -D results shown in Fig. 9. But, the goal here is to determine the fundamental effect of compliant walls on secondary disturbances.

Final amplitude calculations using eqns. (27) are carried out for the frequency $F r \simeq 53$. In Fig. 10, secondary amplitudes arising from the most amplified 3D primary wave over a non-isotropic compliant wall are compared to the previous results from 2-D primary waves over rigid and non-isotropic walls. At this frequency, the amplitude difference between the $2-\mathrm{D}$ and 3 - $\mathrm{D}$ primary instabilites is small, yet the secondary disturbance responds notably. This is an indication that small changes in the primary instability, however slight, have a mounting effect on the rapidly developing secondary instability.

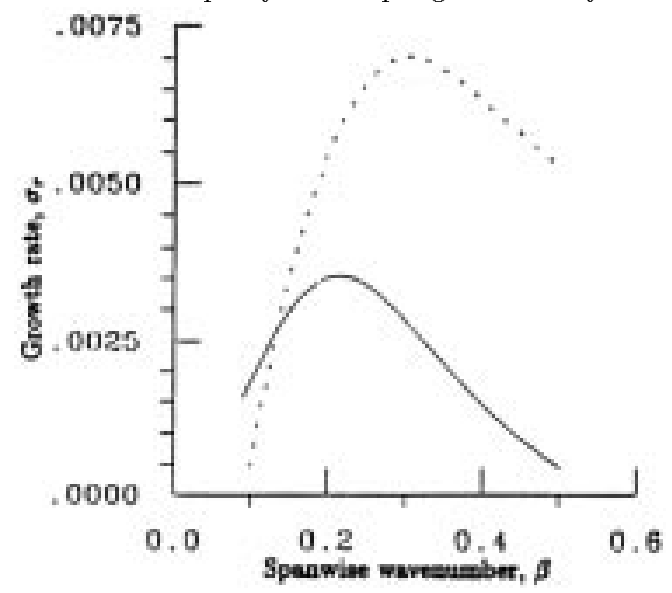

Fig. 9 Growth rates of the subharmonic disturbance for 2-D and 3-D primary waves over the isotropic wall as a function of spanwise wavenumber for $R_{\delta^{*}}=1760$ and $F r \simeq 30.2$ for $\longrightarrow, \phi=0^{\circ}$ with $A=0.010 ; \cdots, \phi=45^{\circ}$ with $A=0.031$.

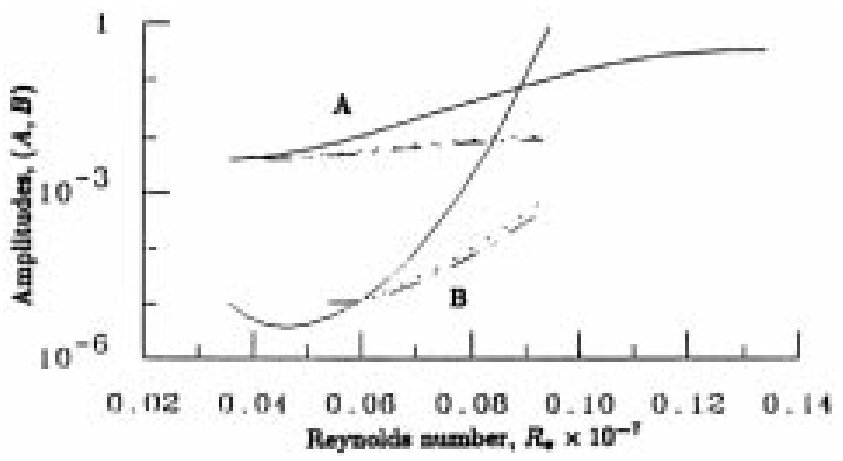

Fig. 10 Amplitude growth as a function of Reynolds number for the subharmonic mode (B) of a $2-\mathbf{D}$ and $3-\mathbf{D}$ primary wave $(\mathbf{A})$ at $F r \simeq 53$, $B_{0}=1 \times 10^{-5}, A_{0}=0.004$, and $\bar{b}=0.15$ over a - , rigid wall $-\cdots-, 2-\mathrm{D}$ non-isotropic wall; and $\cdots$, 3-D non-isotropic wall. 


\section{Summary}

In earlier studies ${ }^{15,16,21}$, it was shown that 3 -D primary instabilities theoretically dominate transition over the compliant walls considered, yet transition delays were found compared to the rigid wall. The present paper has further extended the understanding of the effect of compliant walls on transition mechanisms in boundary layers. Namely, the effect compliant walls have on secondary instabilities has been investigated. It has been shown that the use of compliant walls can lead to reduced growth rates and amplification of secondary instabilities. From both the earlier 3 -D primary results combined with the present secondary findings, it should be emphasized that the physical nature and make-up of the mechanisms in transition are not altered by the control device (i.e. compliant wall). Rather, only the response of that mechanism is changed. This fact is of particular importance for designing Laminar Flow Control (LFC) studies. As an example, the behavior of a secondary instability growth with variation in primary amplitude is well documented by Herbert. As the primary amplitudes are reduced, the excitement of the secondary instability is delayed. Thus, active or passive devices which suppress primary instability growth should lead to corresponding suppression and delay of succeeding instabilities. This has been demonstrated above with the compliant wall. The twofold major discovery and demonstration of the present investigation is: (1) the use of passive devices, such as compliant walls, lead to significant reductions in the secondary instability growth rates and amplification; (2) suppressing the primary growth rates and subsequent amplification enable delays in the growth of the explosive secondary instability mechanism.

\section{Acknowledgments}

Support for this project was supplied by the Naval Sea Systems Command and the Applied Research Laboratory Exploratory and Foundational Research Program under NAVSEA N0039-88-C-0051 at the Department of Aerospace Engineering, The Pennsylvania State University. A grant for computational support was provided by the National Science Foundation with the Pittsburgh Supercomputing Center.

\section{References}

1. Kramer, M.O., "Boundary-Layer Stabilization by Distributed Damping," Journal of the Aeronautical Sciences, Vol. 24, No.6, 1957, pp. 459-460.

2. Kramer, M.O., "Hydrodynamics of the Dolphin," Advances in Hydroscience, Vol. 2, 1965, pp. 111130.

3. Bushnell, D.M., "NASA Research on Viscous Drag Reduction II," Laminar-Turbulent Boundary Layers, Vol. 11, 1984, pp. 93-98.
4. Reischman, M.M., "A Review of Compliant Coating Drag Reduction Research at ONR," LaminarTurbulent Boundary Layers, Vol. 11, 1984, pp. 99105.

5. Bushnell, D.M., Hefner, J.N., and Ash, R.L., "Effect of Compliant Wall Motion on Turbulent Boundary Layers," The Physics of Fluids, Vol. 20, 1977, pp. $\mathrm{S} 31-\mathrm{S} 48$.

6. Riley, J.J., Gad-el-Hak, M., and Metcalfe, R.W., "Compliant Coatings," Annual Review of Fluid Mechanics, Vol. 20, 1988, pp. 393-420.

7. Gad-el-Hak, M., "Compliant Coatings Research: A Guide to the Experimentalist," Journal of Fluids and Structures, Vol. 1, 1987, pp. 55-70.

8. Gad-el-Hak, M., "Boundary Layer Interactions with Compliant Coatings: An Overview," Applied Mechanics Reviews, Vol. 39, 1988, pp. 511-523.

9. Carpenter, P.W., "Status of Transition Delay using Compliant Walls," Viscous Drag Reduction in Boundary Layers, Vol. 123, 1990, pp. 79113, (ed. D.M. Bushnell and J.N. Hefner), AIAA:Washington, D.C.

10. Carpenter, P.W., and Garrad, A.D., "The Hydrodynamic Stability of Flow over Kramer-Type Compliant Surfaces:Part 1. Tollmien-Schlichting Instabilities," Journal of Fluid Mechanics, Vol. 155, 1985, pp. 465-510.

11. Carpenter, P.W., and Garrad, A.D., "The Hydrodynamic Stability of Flow over Kramer-Type Compliant Surfaces:Part 2. Flow-Induced Surface Instabilities," Journal of Fluid Mechanics, Vol. 170, 1986, pp. 199-232.

12. Willis, G.J.K., "Hydrodynamic Stability of Boundary Layers over Compliant Surfaces," Ph.D. Thesis, University of Exeter, 1986.

13. Gaster, M., "Is the Dolphin a Red-Herring?", Turbulence Management and Relaminarisation, 1988, pp. 285-304, (ed. H.W. Liepmann and R. Narasimha), IUTAM:Bangalore, India.

14. Yeo, K.S., "The Stability of Flow over Flexible Surfaces," Ph.D. Thesis, University of Cambridge, 1986.

15. Carpenter, P.W., and Morris, P.J., "Growth of 3D Instabilities in Flow over Compliant Walls," Proceedings of the 4 th A sian Congress of Fluid Mechanics, 1989, Hong Kong.

16. Joslin, R.D., Morris, P.J., and Carpenter, P.W., "The Role of Three-Dimensional Instabilities in Compliant Wall Boundary-Layer Transition," (to appear) AIA A Journal, August 1991. 
17. Grosskreutz, R., "An Attempt to Control Boundary-Layer Turbulence with Non-Isotropic Compliant Walls," University Science Journal Dar es Salaam, Vol. 1, 1975, pp. 65-73.

18. Herbert, Th., "Secondary Instabilities of Boundary Layers," Annual Review of Fluid Mechanics, Vol. 20, 1988, pp. 487-526.

19. Klebanoff, P.S., Tidstrom, K.D., and Sargent, L.M., "The Three-Dimensional Nature of Boundary-Layer Transition," Journal of Fluid Mechanics, Vol. 12, 1962, pp. 1-34.

20. Kachanov, Y.S., and Levchenko, V.Y., "The Resonant Interaction of Disturbances at LaminarTurbulent Transition in a Boundary Layer," Journal of Fluid Mechanics, Vol. 138, 1984, pp. 209-247.

21. Joslin, R.D., "The Effect of Compliant Walls on Three-Dimensional Primary and Secondary Instabilities in Boundary-Layer Transition," Ph.D. Thesis, The Pennsylvania State University, 1990.

22. Carpenter, P.W., and Morris, P.J., "The Effects of Anisotropic Wall Compliance on Boundary-Layer Stability and Transition," Journal of Fluid Mechanics, Vol. 218, 1990, pp. 171-223.

23. Herbert, Th., Bertolotti, F.P., and Santos, G.R., "Floquet Analysis of Secondary Instability in Shear Flows," Stability of Time-Dependent and Spatially Varying Flows, 1985, pp. 43-57, (ed. D.L. Dwoyer and M.Y. Hussaini), Springer-Verlag:New York.

24. Bertolotti, F.P., "Temporal and Spatial Growth of Subharmonic Disturbances in Falkner-Skan Flows," M.S. Thesis, Virginia Polytechnic Institute and State University, 1985.

25. Herbert, Th., "Subharmonic Three-Dimensional Disturbances in Unstable Plane Shear Flows," AIAA Paper 83-1759, July 1983.

26. Herbert, Th., "Analysis of Secondary Instabilities in Boundary-Layers," Proceedings of the 10th U.S. National Congress of Applied Mechanics, The University of Texas at Austin, 1988, (ed. H. Lamb), ASME.

27. Spalart, P.R., and Yang, K.-S., "Numerical Study of Ribbon-Induced Transition in Blasius Flow," Journal of Fluid Mechanics, Vol. 178, 1987, pp. 345-365.

28. Balachandar, S., Streett, C.L., and Malik, M.R., "Secondary Instability in Rotating Disk Flows," AIAA Paper 90-1527, June 1990. 